\title{
Research Article Adaptive S-Method for SAR/ISAR Imaging
}

\author{
LJubiša Stanković, ${ }^{1}$ Thayananthan Thayaparan, ${ }^{2}$ Vesna Popović, ${ }^{1}$ Igor Djurović,, ${ }^{1}$ and Miloš Daković ${ }^{1}$ \\ ${ }^{1}$ Electrical Engineering Department, University of Montenegro, 81000 Podgorica, Montenegro \\ ${ }^{2}$ Radar Applications and Space Technology, Defence Research and Development, Ottawa, Ontario, Canada K1A 0Z4
}

Correspondence should be addressed to Igor Djurović, igordj@cg.ac.yu

Received 8 June 2007; Accepted 8 November 2007

Recommended by Sven Nordholm

\begin{abstract}
We propose the adaptive S-method-based technique for imaging of SAR and ISAR targets. This approach can be applied in the 1D and $2 \mathrm{D}$ modes. It is a postprocessing technique, since the first stage is the standard radar imaging with the 2D Fourier transform. In addition, selection of the adaptive parameter in this technique is efficient and can be performed based on simple rules in real time. The proposed technique produces highly concentrated (focused) radar image without interferences commonly associated with time-frequency representations and without defocusing target images that are already focused in the 2D Fourier domain.
\end{abstract}

Copyright (C 2008 LJubiša Stanković et al. This is an open access article distributed under the Creative Commons Attribution License, which permits unrestricted use, distribution, and reproduction in any medium, provided the original work is properly cited.

\section{INTRODUCTION}

When radar transmits an electromagnetic signal to a target, the signal reflects from it and returns to radar. The reflected signal, as compared to the transmitted signal, is delayed, changed in amplitude, and possibly shifted in frequency. These parameters of the received signal contain information about the target's characteristics. For example, delay is related to the target's distance from the radar. The synthetic aperture radar (SAR) and the inverse synthetic aperture radar (ISAR) are systems for obtaining high-resolution image of a target based on the changes in viewing angle of the target with respect to the radar. Relative motion between radar and target produces these viewing angle changes. In the case of ISAR, radar is fixed while the target is moving, while in the SAR case radar is carried on an aircraft or spacecraft platform, moving at uniform speed and constant altitude [1].

Common technique for SAR and ISAR imaging is based on the 2D Fourier transform (FT). This technique is appropriate for imaging nonmoving SAR targets and for rigid body imaging of ISAR targets [1-4]. However, for fast maneuvering ISAR targets $[1,5]$ and targets with $3 \mathrm{D}$ motion $[1,6]$, radar image can be spread in the $2 \mathrm{D}$ FT domain. Similar defocusing effects are observed in the SAR systems for moving targets $[7,8]$. In addition, image of moving targets in SAR systems can be dislocated from the proper position $[1,7,8]$. An additional problem in both SAR and ISAR systems is the micro-Doppler effect caused by fast rotation and vibration of the radar target parts $[9,10]$. Thus, some more sophisticated techniques should be employed for focusing radar images. One group of techniques is based on the motion compensation. These techniques may be based on direct estimation of the target motion parameters, or these parameters can be extracted indirectly by estimating parameters of received signals. Excellent results can be achieved using these techniques, but at the expense of the high computational load [11-15].

An alternative group of techniques is based on the timefrequency $(\mathrm{TF})$ representations $[1,10,16]$. Some of the most common TF representations, such as the Wigner distribution (WD), produce highly concentrated signal terms but with drawback in appearance of undesired interference (cross) terms [17-19]. The S-method (SM), as a TF representation that can produce significant improvement in imaging of radar targets (as in the WD) without introducing cross-terms (as in the standard 2D FT technique), is proposed recently [20]. This method has a parameter that represents a window width in the frequency domain [21]. Results achieved by the SM, although computational simple, may depend on this parameter. Namely, for a very narrow window the obtained radar image could still be spread, while for a very wide window the obtained image can be corrupted by crossterms. In this paper, we propose a technique for adaptive selection of the window width in the SM. This technique is very effective. It is based on simple rules and brings major advantages: moderate calculational burden with highly concentrated radar images without interference terms. 1D and 
2D forms of the adaptive SM are proposed with implementation issues discussed. An important step in the adaptive SM calculation is threshold determination. The threshold value can be determined in various manners. The Otsu algorithm [22] based procedure for automatic threshold determination is used here. The proposed technique is tested on several examples of the SAR and ISAR images. Note that special purpose hardware for both $1 \mathrm{D}$ and 2D adaptive SM is proposed and analyzed in details recently in [23]. All these facts confirm that this is a very promising technique for both SAR and ISAR imaging.

The manuscript is organized as follows. Brief review of the signal model in considered systems is given in Section 2 along with standard imaging techniques. The proposed technique is presented in Section 3. Results of simulations are given in Section 4.

\section{SIGNAL MODEL}

In both SAR and ISAR systems series of signals is transmitted toward radar target. Commonly, these signals are chirps (linear frequency modulated (FM) signals), but some other waveforms are also used in practice. After receiving, these signals are demodulated to the baseband with possible distance compensation and some other preprocessing operations (such as pulse compression). Let the preprocessed received signal be denoted as $q(m, t)$, where $t$ is the time index (so-called fast-time coordinate) while $m \in[0, M)$ corresponds to the signal number transmitted toward a target (socalled slow-time coordinate). Commonly, for simpler processing, signal is sampled in the fast-time with the properly selected sampling interval $q(m, n)=q(m, n \Delta t)$.

The received signal for point scatterer model $[1,6]$ can be presented as a sum of the FM signals:

$$
q(m, n)=\sum_{i} \sigma_{i} \exp \left(j \phi_{i}(m, n)\right),
$$

where $\sigma_{i}$ is reflection coefficient of the corresponding scatterer. Form of the phase functions $\phi_{i}(m, n)$ depends on the type of the corresponding radar scatterer. Here, we will give approximative forms of the phase function for some typical cases in both SAR and ISAR systems.

(1) For nonmoving targets in SAR systems and for constant velocity targets in ISAR systems, the phase function can be approximated by:

$$
\phi_{i}(m, n)=a_{i}^{(1)} m+b_{i}^{(1)} n .
$$

So, the received signal is a $2 \mathrm{D}$ complex sinusoid.

(2) Phase function of moving targets in the SAR systems is analyzed in [1]. Similar results are observed in some ISAR systems with uniform acceleration of targets:

$$
\phi_{i}(m, n)=a_{i}^{(2)} m^{2} / 2+a_{i}^{(1)} m+b_{i}^{(1)} n
$$

Corresponding signal is the linear FM one along $m$ and complex sinusoid along $n$.

(3) Recent surveys in $[24,25]$ have shown that returns from nonuniform moving targets in the SAR systems can be accurately represented only with higher-order polynomial phase FM signals:

$$
\phi_{i}(m, n)=\sum_{p=1}^{P} a_{i}^{(p)} \frac{m^{p}}{p !}+b_{i}^{(1)} n,
$$

where $P>2$.

(4) ISAR targets with fast and 3D maneuvers can produce phase function of the form

$$
\phi_{i}(m, n)=a_{i}^{(1)} m+b_{i}^{(1)} n+\sum_{p=1}^{P} \sum_{k=1}^{K} d_{i}^{(p, k)} \frac{m^{p}}{p !} \frac{n^{k}}{k !}
$$

where parameters in slow and fast-time cannot be treated as independent like in the previous cases.

(5) For fast rotating or vibrating parts of the SAR/ISAR target, the phase function can be modeled as a sinusoidally modulated FM signal:

$$
\phi_{i}(m, n)=a_{i}^{(1)} m+b_{i}^{(1)} n+c_{i} \sin \left(\alpha_{i} m+\beta_{i} n+\varphi_{i}\right) .
$$

In all considered cases, the phase functions can be written as $\phi_{i}(m, n)=a_{i}^{(1)} m+b_{i}^{(1)} n+\psi_{i}(m, n)$, where $\psi_{i}(m, n)$ represents higher-order terms in the signal phase, while parameters $\left(a_{i}^{(1)}, b_{i}^{(1)}\right)$ correspond to the position of scatterers. Other introduced parameters $a_{i}^{(p)}, d_{i}^{(p, k)}, c_{i}, \alpha_{i}, \beta_{i}$, and $\varphi_{i}$ depend on the position of the targets, relative motion, radar systems parameters, parameters of the target motion, and some other effects.

The radar image can be obtained by using the $2 \mathrm{D} \mathrm{FT}^{1}$ as:

$$
\begin{aligned}
Q\left(m^{\prime}, n^{\prime}\right)= & \sum_{m} \sum_{n} q(m, n) w(m, n) \\
& \times \exp \left(-j 2 \pi m m^{\prime} / M-j 2 \pi n n^{\prime} / N\right),
\end{aligned}
$$

where $N$ is the number of samples in the fast-time direction, while $w(m, n)$ is a window function used to reduce spectral leakage effects in the FT domain. For a single scatterer return that corresponds to the nonmoving targets in the SAR systems and rigid body parts in the ISAR systems (case 1) the 2D FT is

$$
Q_{i}\left(m^{\prime}, n^{\prime}\right)=\sigma_{i} W\left(m^{\prime}-M a_{i}^{(1)} / 2 \pi, n^{\prime}-N b_{i}^{(1)} / 2 \pi\right),
$$

where $W(\cdot)$ is 2D FT of the window function. Since a window is commonly designed to be highly concentrated in the FT domain, we can assume that, for stationary targets, radar image is highly concentrated around position that is proportional to $\left(a_{i}^{(1)}, b_{i}^{(1)}\right)$, and these parameters are proportional to the position of the scatterer point.

For other forms of the phase function the 2D FT can be represented in the following form:

$$
\begin{aligned}
Q_{i}\left(m^{\prime}, n^{\prime}\right)= & \sigma_{i} W\left(m^{\prime}-M a_{i}^{(1)} / 2 \pi, n^{\prime}-N b_{i}^{(1)} / 2 \pi\right) \\
& *_{m^{\prime}} *_{n^{\prime}} \operatorname{FT}\left\{\exp \left(j \psi_{i}(m, n)\right)\right\},
\end{aligned}
$$

\footnotetext{
${ }^{1}$ Recently, backprojection techniques are used for the SAR imaging. Since all the basic phenomena in the received signal are the same as in the $2 \mathrm{D}$ FT imaging, we will here consider the simpler 2D FT technique.
} 
where $*_{m^{\prime}} *_{n^{\prime}}$ represents $2 \mathrm{D}$ convolution, while the term $\mathrm{FT}\left\{\exp \left(j \psi_{i}(m, n)\right)\right\}$ causes spreading and possible dislocating component from the proper position. The motion compensation techniques compensate this term based on the estimation of motion parameters of the targets. Alternatively, it can be performed by estimating higher-order coefficients in the signal phase [11-15]. However, these techniques are very computationally demanding. The TF representations are another approach that will be considered in the next section.

\section{RADAR IMAGING BY USING TF REPRESENTATIONS}

\subsection{Background}

It has already been shown that the imaging based on the 2D FT causes spreading of radar images. In order to avoid complex valued nature of the $2 \mathrm{D} \mathrm{FT}$, its squared magnitude $\left|Q\left(m^{\prime}, n^{\prime}\right)\right|^{2}$ is commonly used for imaging that is equivalent to the periodogram in spectral analysis (or to the spectrogram in the TF analysis). In the TF analysis, the WD is a tool that can be used to improve concentration of the radar image, that is, to reduce spreading. However, the WD has a serious drawback in the form of appearance of spurious components called cross-terms. The cross-terms may be so emphatic that they mask the useful components. Then, design of the TF representations that have more concentrated components than in the standard image but without undesired effects is the goal of this paper.

\section{2. $S M$}

The SM is technique widely used in the TF analysis for cross-terms free TF representation (or representation with reduced cross-terms) giving highly concentrated TF components [26]. The SM can be defined for radar images as [20]

$$
\operatorname{SM}_{1}\left(m^{\prime}, n^{\prime}\right)=\sum_{k} \Pi(k) Q\left(m^{\prime}+k, n^{\prime}\right) Q^{*}\left(m^{\prime}-k, n^{\prime}\right),
$$

where $\Pi(k)$ is window in the frequency domain. For fixed $n^{\prime}$ this form is the 1D SM for fixed-range cell. In similar manner, radar image based on the 1D SM for fixed cross-range cell is [20]

$$
\mathrm{SM}_{2}\left(m^{\prime}, n^{\prime}\right)=\sum_{l} \Pi(l) Q\left(m^{\prime}, n^{\prime}+l\right) Q^{*}\left(m^{\prime}, n^{\prime}-1\right) .
$$

Also, the 2D SM is [20]

$$
\begin{aligned}
\mathrm{SM}_{3}\left(m^{\prime}, n^{\prime}\right)= & \sum_{k} \sum_{l} \Pi(k, l) Q\left(m^{\prime}+k, n^{\prime}+l\right) \\
& \times Q^{*}\left(m^{\prime}-k, n^{\prime}-l\right) .
\end{aligned}
$$

Commonly, the frequency window function is rectangular, and for 1D SM given with (10) exhibits

$$
\Pi(k)= \begin{cases}1, & |k| \leq K \\ 0, & \text { elsewhere }\end{cases}
$$

Then, the corresponding SM form can be calculated as

$$
\begin{aligned}
& \operatorname{SM}_{1}\left(m^{\prime}, n^{\prime}\right) \\
& =\left|Q\left(m^{\prime}, n^{\prime}\right)\right|^{2}+2 \operatorname{Re}\left\{\sum_{k=1}^{K} Q\left(m^{\prime}+k, n^{\prime}\right) Q^{*}\left(m^{\prime}-k, n^{\prime}\right)\right\} .
\end{aligned}
$$

For $K=0$ we obtain the standard radar image (defocused but without spurious terms), while for large $K$ the radar image approaches the WD-based image (focused but with interfering cross-terms). Fortunately, for relatively small $K$ the radar image could be significantly improved without introducing the interference. A drawback of this technique is demonstrated in the next subsection on a simple example.

\subsection{Illustrative example}

Consider three point targets. The first and third targets are moving with constant velocity in SAR (equivalent to the uniform acceleration in ISAR), while the second is nonmoving target in SAR (equivalent to the constant velocity in ISAR). The returned radar signal from these three targets can be modelled as (Section 2)

$$
\begin{aligned}
x(n)= & A(n) e^{-j(0.4 / 256) \pi n^{2}} e^{-j(\pi / 2) n}+A(n) e^{j(\pi / 8) n} \\
& +A(n) e^{j(0.2 / 256) \pi n^{2}} e^{j(\pi / 3) n},
\end{aligned}
$$

where the amplitude $A(n)$ is slow-varying, defined as $A(n)=$ $1 / 2+(1 / 2) \cos (2 \pi / 256) n$. The signal is observed for $-128 \leq$ $n \leq 127$. For the considered time instant $n=0$, it will be $\omega_{1}(0)=-\pi / 2, \omega_{2}(0)=\pi / 8$, and $\omega_{3}(0)=\pi / 3$.

The spectrogram of the analyzed signal is shown in Figure 1(a). The first and third targets are spread and not visible compared to the second target. Note that the radar signal returned from the second target is constant-frequency component, while the radar signals returned from the first and third targets are linear FM.

The spectrogram corresponds to the SM with $K=0$. By increasing the value of $K$ concentration of the first and third targets is improved, Figure 1 (b) $K=4$, but these targets are still spread compared to the second one. For higher value of $K, K=16$, concentration of the components continue to increase, but the cross-term appears between close targets, the second and third in Figure 1(c). The desired concentration of the first and third components is achieved by using the WD, SM with $K$ equal to the signal length, but this value generates very strong cross-terms between components, see Figure 1(d). The cross-term between the second and third component is almost strong as useful components. Thus, it is clear that we need a more sophisticated 


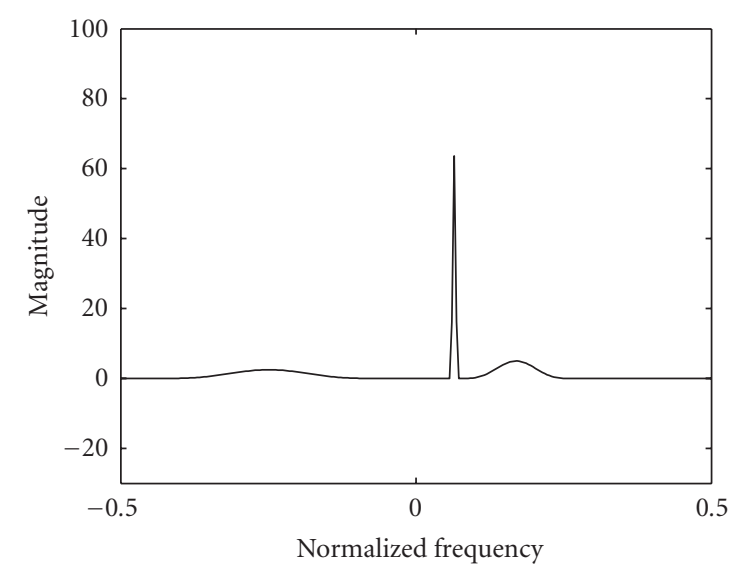

(a)

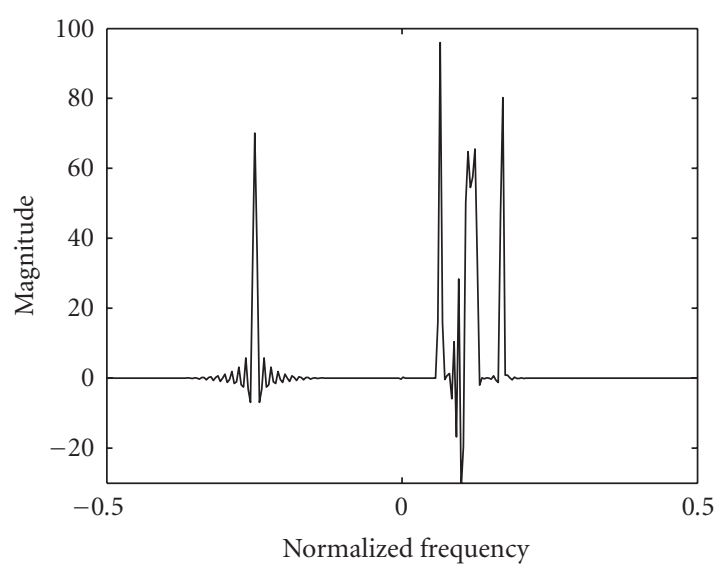

(c)

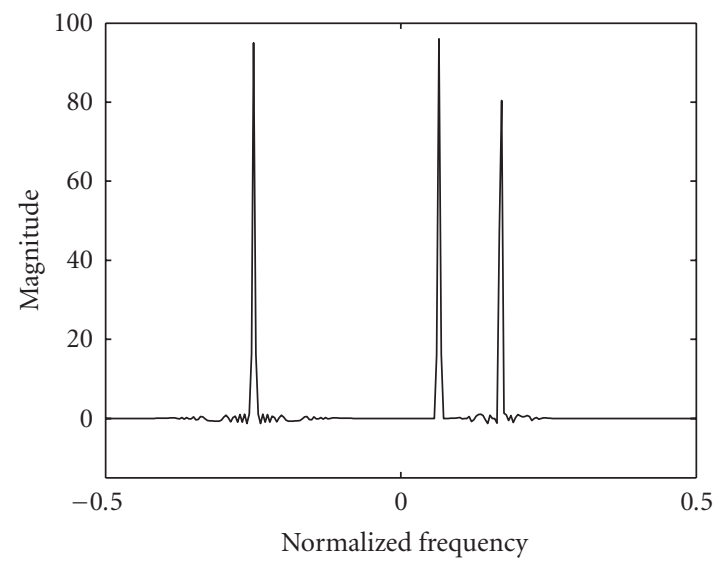

(e)

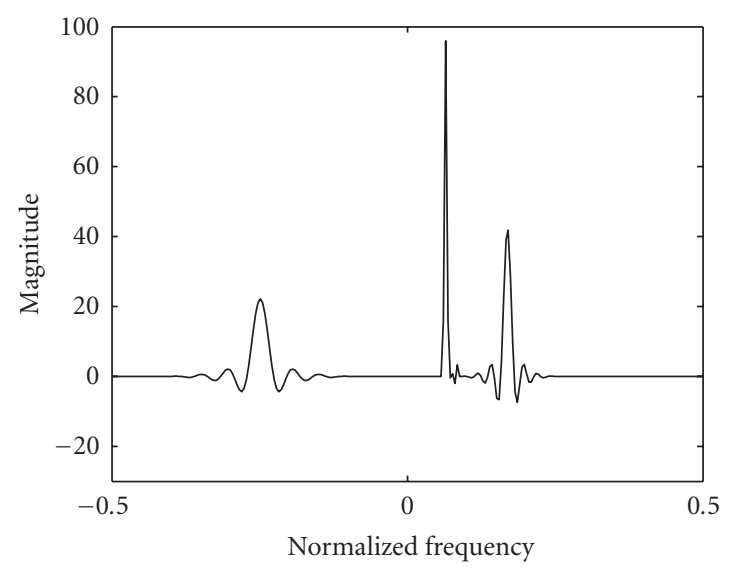

(b)

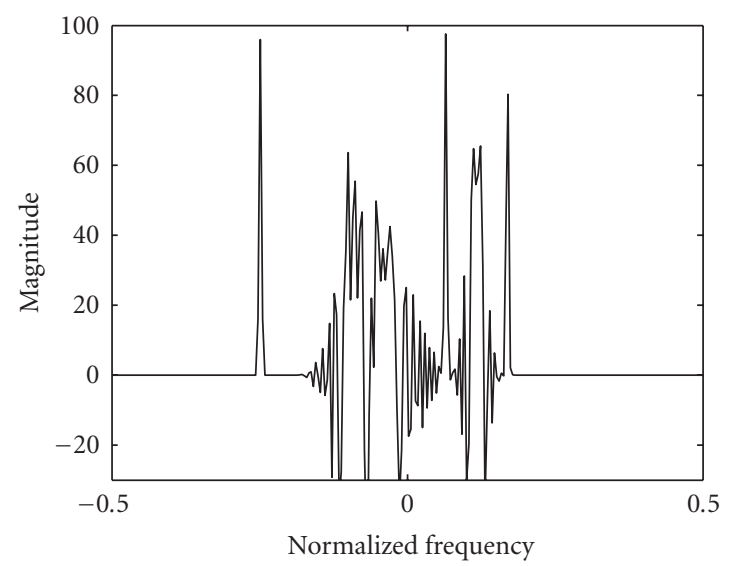

(d)

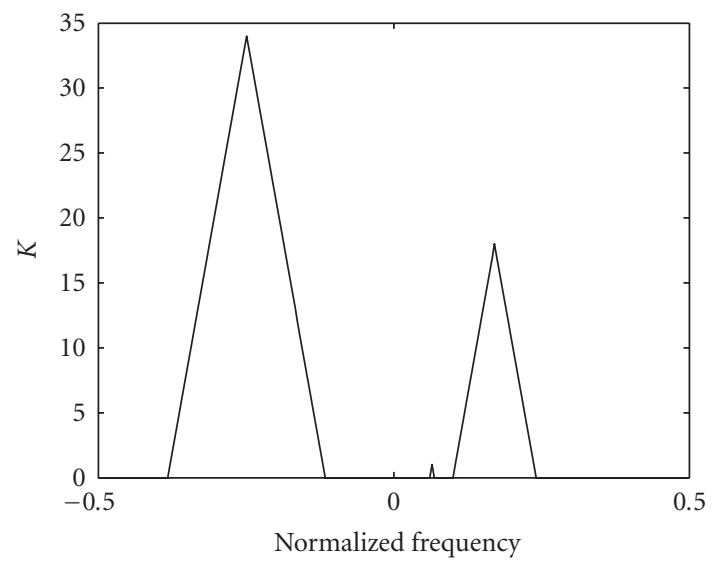

(f)

FIGURE 1: Representation of the three-component signal by using: (a) spectrogram (S-method with $K=0$ ), (b) S-method with $K=4$, (c) S-method with $K=16$, (d) Wigner distribution (S-method with $K$ equal to the signal length), and (e) adaptive S-method. (f) Values of $K$ used for obtaining the adaptive S-method.

technique for radar imaging in order to achieve high concentration without interferences. Fortunately, one possible solution, the adaptive $\mathrm{SM}$, is quite simple. The resulting representation of the analyzed signal is given in Figure 1(e). Here, we assumed that value of $K$ depends on the considered frequency. It can be observed that high values of
$K$ are used where concentration improvement is necessary (around $\omega_{1}(0)$ and $\omega_{3}(0)$ ), while in the case when the component is well concentrated in the spectrogram, very low values of $K$ (or $K=0$ ) are used. In the next subsection selection of the adaptive window in real problems is discussed. 


\subsection{Adaptive 1D SM}

Here, we give a form of the adaptive SM for fixed-range cell, but in the same manner it can be evaluated for fixed crossrange cell. The adaptive SM is originally developed for improving the TF representation in [21].

The adaptive 1D SM for radar images can be defined as

$$
\begin{aligned}
& \operatorname{SM}_{1}\left(m^{\prime}, n^{\prime}\right) \\
& =\left|Q\left(m^{\prime}, n^{\prime}\right)\right|^{2}+2 \operatorname{Re}\left\{\sum_{k=1}^{K\left(m^{\prime}, n^{\prime}\right)} Q\left(m^{\prime}+k, n^{\prime}\right) Q^{*}\left(m^{\prime}-k, n^{\prime}\right)\right\} .
\end{aligned}
$$

The main problem here is determination of $K\left(m^{\prime}, n^{\prime}\right)$. $K\left(m^{\prime}, n^{\prime}\right)$ can be simply obtained as a maximal value of $k$ for which the term $\operatorname{Re}\left\{Q\left(m^{\prime}+k, n^{\prime}\right) Q^{*}\left(m^{\prime}-k, n^{\prime}\right)\right\}$ used for the SM calculation is greater than a specific threshold $R\left(m^{\prime}, n^{\prime}\right)$, and where all $\operatorname{Re}\left\{Q\left(m^{\prime}+k^{\prime}, n^{\prime}\right) Q^{*}\left(m^{\prime}-k^{\prime}, n^{\prime}\right)\right\}$ for $\left|k^{\prime}\right|<|k|$ are greater than the threshold. This can be written as

$K\left(m^{\prime}, n^{\prime}\right)$

$=\arg \max _{k} \bigwedge_{k^{\prime}=1}^{k}\left(\operatorname{Re}\left\{Q\left(m^{\prime}+k^{\prime}, n^{\prime}\right) Q^{*}\left(m^{\prime}-k^{\prime}, n^{\prime}\right)\right\} \geq R\left(m^{\prime}, n^{\prime}\right)\right)$,

where $\wedge_{k^{\prime}=1}^{k}$ represents logical operation AND applied to logical expressions from argument for various $k^{\prime}=1, \ldots, k$.

The threshold value can be determined in various ways. The global threshold is proposed in [21] as

$$
R=\varepsilon \max _{m^{\prime}, n^{\prime}}\left|Q\left(m^{\prime}, n^{\prime}\right)\right|^{2},
$$

where $\varepsilon$ can be adopted as a small value, for example, $\varepsilon \in$ $[0.1 \%, 5 \%]$. Of course, this threshold can be calculated in the same manner for considered range or cross-range cell. In this way components having small energy are removed and they are indication of the end of the useful radar component. In the case of images corrupted by a noise we can select global threshold as

$$
R=\max \left\{\varepsilon \max _{m^{\prime}, n^{\prime}}\left|Q\left(m^{\prime}, n^{\prime}\right)\right|^{2}, \kappa^{2} \sigma^{2}\left(m^{\prime}, n^{\prime}\right)\right\},
$$

where $\sigma^{2}\left(m^{\prime}, n^{\prime}\right)$ is variance of the radar image caused by the noise. An analysis of noise in the SM can be found in [27]. In this case we can remove all weak components, as well as the components influenced by noise. Parameter $\kappa$ is commonly selected to be around $\kappa=3$ (three sigma rule).

This thresholding approach can be applied locally for regions of the radar image. In addition, well-described techniques from the digital image processing can be used in this application [22]. The procedure for the threshold determination based on the Otsu algorithm [22, pages 598-600] is used here. The 2D FT magnitude $\left|Q\left(m^{\prime}, n^{\prime}\right)\right|$ is taken as image pixels intensity needed for the algorithm.

Step 1. Estimate initial value for threshold. Here, initial value for threshold is set to the half of the pixels intensity maximum:

$$
\rho=\frac{1}{2} \max _{m^{\prime}, n^{\prime}}\left|Q\left(m^{\prime}, n^{\prime}\right)\right|
$$

Step 2. Calculate two sums $S_{1}$ and $S_{2}$, where $S_{1}$ is a sum of intensity values of the pixels whose intensity is larger than the current threshold $\rho$ :

$$
S_{1}=\sum_{m^{\prime}, n^{\prime}}\left|Q\left(m^{\prime}, n^{\prime}\right)\right| \quad\left(\forall\left(m^{\prime}, n^{\prime}\right),\left|Q\left(m^{\prime}, n^{\prime}\right)\right|>\rho\right),
$$

while $S_{2}$ is a sum of intensity values of the pixels whose intensity is smaller than the current threshold $\rho$ :

$$
S_{2}=\sum_{m^{\prime}, n^{\prime}}\left|Q\left(m^{\prime}, n^{\prime}\right)\right| \quad\left(\forall\left(m^{\prime}, n^{\prime}\right),\left|Q\left(m^{\prime}, n^{\prime}\right)\right|<\rho\right) .
$$

Step 3. Calculate two new thresholds, $\rho_{1}$ and $\rho_{2}$, as average values of the obtained sums:

$$
\rho_{1}=\frac{S_{1}}{N_{1}}, \quad \rho_{2}=\frac{S_{2}}{N_{2}},
$$

where $N_{1}$ and $N_{2}$ are number of elements summed in (21) and (22), respectively.

Step 4. Compute a new threshold value

$$
\rho=\frac{1}{2}\left(\rho_{1}+\rho_{2}\right)
$$

Step 5. Repeat Steps 2 through 4 until the difference in $\rho$ in successive iterations is smaller than a predefined parameter, or for a specified number of iteration.

The thresholds used in our simulations are obtained after five iterations. Its squared value, $R=\rho^{2}$, is used in both $1 \mathrm{D}$ and $2 \mathrm{D}$ adaptive SM calculation.

\subsection{Adaptive 2D SM}

The adaptive 2D SM can be evaluated as [28]

$$
\begin{aligned}
& \mathrm{SM}_{3}\left(m^{\prime}, n^{\prime}\right) \\
& =\left|Q\left(m^{\prime}, n^{\prime}\right)\right|^{2} \\
& +2 \operatorname{Re}\left\{\sum_{k=0}^{K\left(m^{\prime}, n^{\prime}\right)} \sum_{l=1}^{L\left(m^{\prime}, n^{\prime}\right)} Q\left(m^{\prime}+k, n^{\prime}+l\right) Q^{*}\left(m^{\prime}-k, n^{\prime}-l\right)\right\} \\
& +2 \operatorname{Re}\left\{\sum_{k=1}^{K\left(m^{\prime}, n^{\prime}\right)} \sum_{l=-L\left(m^{\prime}, n^{\prime}\right)}^{0} Q\left(m^{\prime}+k, n^{\prime}+l\right) Q^{*}\left(m^{\prime}-k, n^{\prime}-l\right)\right\} .
\end{aligned}
$$

Here, we have to determine two adaptive values $K\left(m^{\prime}, n^{\prime}\right)$ and $L\left(m^{\prime}, n^{\prime}\right)$ for each pixel in the radar image. However, multiparameter optimization in this case is difficult and it could lead to nonoptimal solutions. Instead, we will use $I\left(m^{\prime}, n^{\prime}\right)=K\left(m^{\prime}, n^{\prime}\right)=L\left(m^{\prime}, n^{\prime}\right)$, so just one adaptive value for width of the used square window has to be determined. The adaptive square window width $I\left(m^{\prime}, n^{\prime}\right)=I$ for the considered radar image pixel can be determined as a maximal value of $I$ for which all terms $Q\left(m^{\prime}+k, n^{\prime}+\right.$ $l) Q^{*}\left(m^{\prime}-k, n^{\prime}-l\right)$ for $|k, l| \leq I$ (inside and at the border of 
TABLE 1: The motion parameters for the targets used in the SAR example.

\begin{tabular}{lccccccc}
\hline Scatterer no. & 1 & 2 & 3 & 4 & 5 & 6 & 7 \\
\hline$x_{0}[\mathrm{~m}]$ & -34 & 0 & 34 & -34 & 34 & -34 & 0 \\
$y_{0}[\mathrm{~m}]$ & 120 & 120 & 120 & 0 & 0 & -120 & -120 \\
$v_{x}\left[\frac{\mathrm{m}}{\mathrm{s}}\right]$ & -25 & 0 & -30 & 0 & 40 & 0 & 0 \\
$v_{y}\left[\frac{\mathrm{m}}{\mathrm{s}}\right]$ & 40 & 30 & -30 & 60 & 0 & 20 & 30 \\
$a_{x}\left[\frac{\mathrm{m}}{\mathrm{s}^{2}}\right]$ & 0 & 0 & 2 & 0 & 0 & 0 & 0 \\
$a_{y}\left[\frac{\mathrm{m}}{\mathrm{s}^{2}}\right]$ & 1 & 0 & 0 & 0 & 0 & 0 & 0 \\
\hline
\end{tabular}

the used square window) are greater than a specific threshold $R\left(m^{\prime}, n^{\prime}\right)$. This can be written as

$$
\begin{aligned}
I\left(m^{\prime}, n^{\prime}\right) & =\arg \max _{I} \bigwedge_{k, l=1}^{I}\left(Q\left(m^{\prime}+k, n^{\prime}+l\right) Q^{*}\left(m^{\prime}-k, n^{\prime}-l\right)\right. \\
& \left.\geq R\left(m^{\prime}, n^{\prime}\right)\right) .
\end{aligned}
$$

The adaptive 2D SM can improve concentration of the radar image using information from both the range and crossrange cells and it can be useful in the case of images with significant spreading in both directions. This spreading can occur in the case of very complicated maneuvers or in the case when radar and target are relatively close to each other, and also in some other setups.

Now, we overview all advantages of the proposed techniques. The adaptive $\mathrm{SM}$ is a postprocessing technique that modifies standard radar image calculated by using the 2D FT. Additional processing consists of two parts: threshold evaluation and adding terms to the standard radar image. Both these steps require just a moderate calculation burden since they consist of simple multiplications, additions, and logical operations. In addition, hardware realization of the SM is well developed for both 1D and 2D signals [23]. This hardware requires just a moderate modification to be used in the proposed application. As it will be seen from Section 4, achieved results with the SM are quite accurate and we see this approach as one of the main candidates to be used as a trade-off between accuracy and quality of radar images and computational demands.

\section{EXAMPLES}

In this section we illustrate the advantages of the proposed technique on the two examples for SAR and ISAR images obtained by using simulated setups. Application of the adaptive 1D SM to the MIG target model is also illustrated at the end of the section.

\subsection{SAR example}

The eight targets setup, where each target can be modeled as point scatterer, is considered in this example. Radar signal reflected from the 8 point scatterers can be obtained by using superposition principle as a sum of individual echoes.
All targets are moving. The position of the radar targets can be described as $x_{i}(t)=x_{i 0}+v_{x i}(0) t+(1 / 2) a_{x i} t^{2}$ and $y_{i}(t)=y_{i 0}+v_{y i}(0) t+(1 / 2) a_{y i} t^{2}$, where the motion parameters are given in Table 1 .

The radar parameters of the Environment Canada's airborne CV 580 SAR system described in [24] are used as a basis for this example. The radar operates at the frequency $f_{0}=5.3 \mathrm{GHz}$, which corresponds to the frequency of C-band of the CV 580 SAR system. The bandwidth of linear FM signals is $B=50 \mathrm{MHz}$, the pulse repetition time is $T=1 / 300 \mathrm{~s}$, with $M=256$ pulses in one revisit. Number of samples within one pulse is $N=256$. The aircraft with a radar is moving along $x$-axis with velocity $V=130 \mathrm{~m} / \mathrm{s}$. Radar altitude is $h=6 \mathrm{~km}$, while radar ground distance to the target is $9400 \mathrm{~m}$.

The conventional SAR image is obtained by 2D FT processing and it is shown in Figure 2(a). Since all targets are moving, the obtained radar image is blurred, while some of the targets are also dislocated from the true position. By applying the signal independent 1D SM-based postprocessing of the obtained 2D FT, the resulting radar image will be more focused, Figure 2(b). By increasing the value of $K$ (in this example $K=8$ is used), the improved concentration is obtained. The 1D SM will produce good performance in the case when the targets are not very close. In the case of close targets (no. 1 and no. 2, and no. 6 and no. 7), the undesired cross-terms will appear between them as a result of its mutual influence. The SAR image obtained by using the adaptive 1D SM will result in the well-concentrated targets, and without undesired cross-terms, see Figure 2(c). Here, the SM and the adaptive SM are calculated along the cross-range. Since target velocities induce spreading in the cross-range, focusing along this axis is needed. Spreading along range direction can also appear in conventionally processed radar image, see Figure 2(a). This spreading appears as a result of fast moving targets and radar setup. In this case, the adaptive 2D SM should be used. Namely, information from both range and cross-range cells are used for adaptive 2D SM calculation. Achieved results are shown in Figure 2(d). The targets in the SAR image obtained by applying the adaptive $2 \mathrm{D}$ SM are better concentrated than the targets in the adaptive 1D SM-based image. The threshold obtained by performing the previously described Otsu algorithm-based procedure is used for the adaptive 2D SM and adaptive 1D SM calculation. 


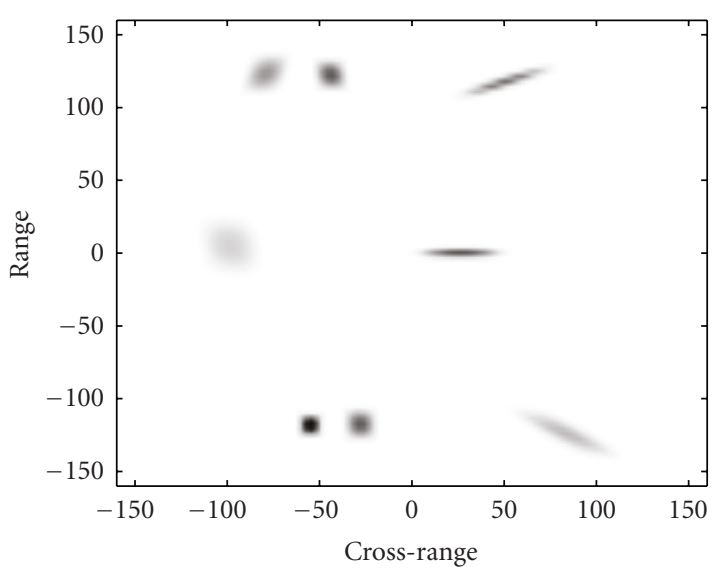

(a)

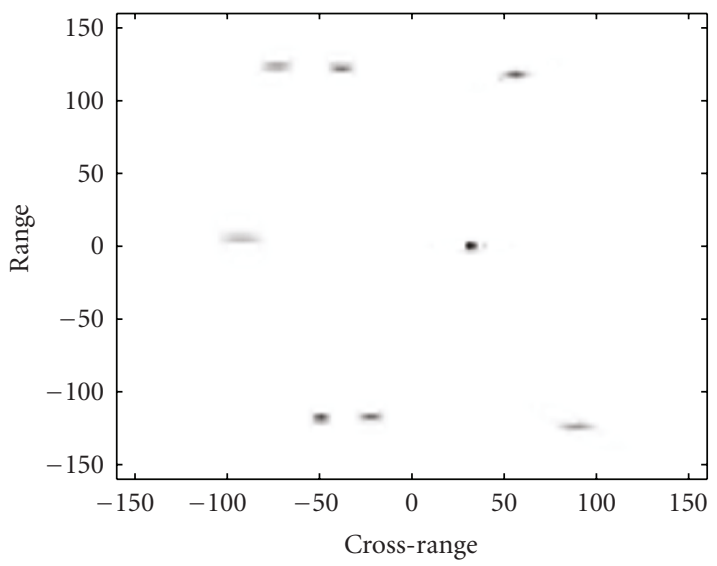

(c)

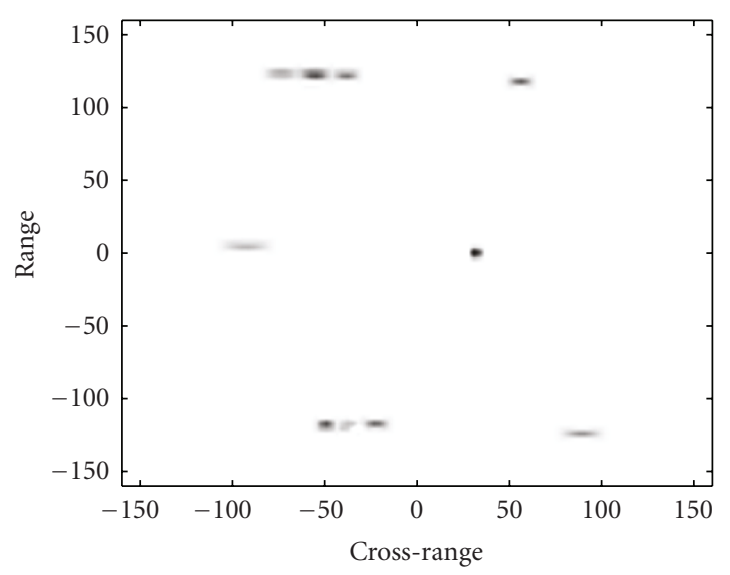

(b)

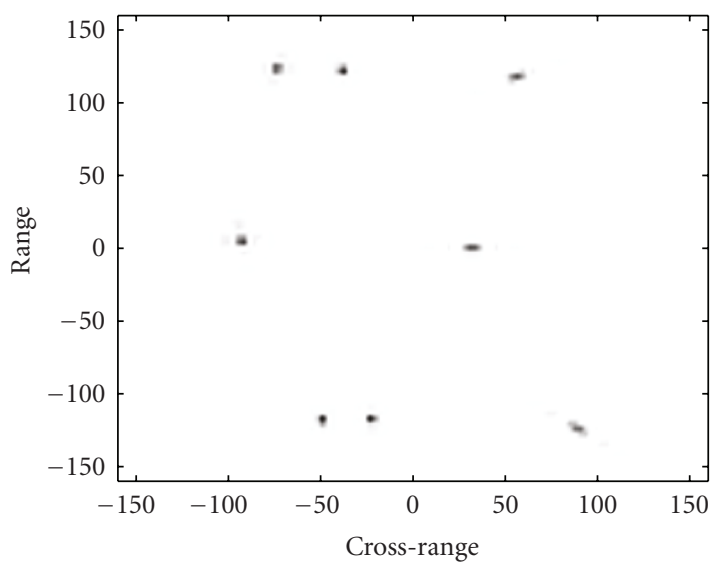

(d)

FIgURE 2: Simulated SAR image obtained by using (a) 2D FT, (b) 1 D SM with $K=8$, (c) adaptive 1D SM, and (d) adaptive 2D SM.

TABLE 2: The positions of the reflectors used for the ISAR target simulation.

\begin{tabular}{lcccccrr}
\hline Reflector No. & 1 & 2 & 3 & 4 & 5 & 6 & \multicolumn{1}{c}{. } \\
\hline$x[\mathrm{~m}]$ & -3.4642 & 0 & 3.4642 & 1.7320 & -1.7320 & 0 & 1.5541 \\
$y[\mathrm{~m}]$ & 2.3094 & 2.3094 & 2.3094 & -1.1547 & -1.1547 & -4.6189 & 0.6414 \\
\hline
\end{tabular}

\subsection{ISAR example}

The radar setup used in [3] is considered as a basis for this example. The high-resolution radar operates at the frequency $f_{0}=10.1 \mathrm{GHz}$, the bandwidth of linear FM signals is $B=1410 \mathrm{MHz}$, the coherent integration time is $T=2 \mathrm{~s}$, with $M=128$ pulses in one revisit. The number of samples within one pulse is $N=128$. The target consisted of seven reflectors is at $R=2 \mathrm{~km}$ distance from the radar, and rotates at $\omega_{R}=4^{\circ} / \mathrm{sec}$. The nonuniform rotation with frequency $\Omega=1 \mathrm{~Hz}, \omega_{R}(t)=\omega_{R}+A \sin (2 \pi \Omega t)$, and amplitude $A=1 \%$ sec is superimposed for each reflector. In addition, all reflectors have uncompensated range velocity $v_{x}=1 \mathrm{~m} / \mathrm{s}$. The distance between radar and targets after range compensation is

$$
d_{i}(t)=x_{i} \cos \left(\theta_{R}(t)\right)+y_{i} \sin \left(\theta_{R}(t)\right)+v_{x} t, \quad i=1, \ldots, 7,
$$

where $\theta_{R}(t)=\omega_{R} t-A /(2 \pi \Omega) \cos (2 \pi \Omega t)$. The positions for each reflector at the beginning of the observation interval are given in Table 2 .

The ISAR images obtained by different radar signal processing methods are shown in Figure 3. The 2D FT will result in blurred image, see Figure 3(a). Improved concentration is obtained by using the 1D SM, see Figure 3(b). Here, $K=8$ is used for the 1D SM calculation. Since some of the reflectors are very close, the cross-terms will appear before obtaining satisfying concentration. The adaptive 1D SM is used in order to avoid cross-terms, while achieving good concentration, see Figure 3(c). It can be seen from Figure 3(a) that the target is also spread along the range direction. This spreading appears as a result of nonuniform rotation performed by the target and radar setup. By applying the 1D SM, as well as the adaptive 1D SM, the significant improvement in concentration is achieved. For improving concentration by 


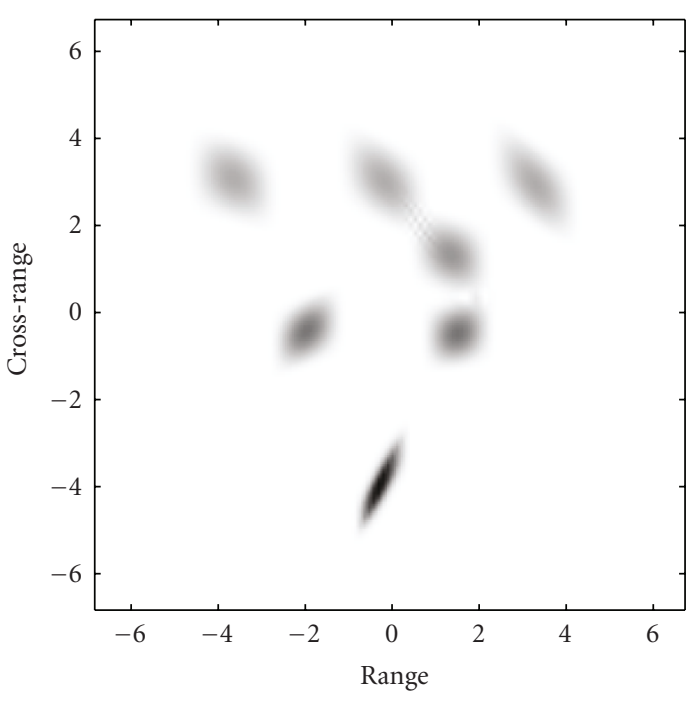

(a)

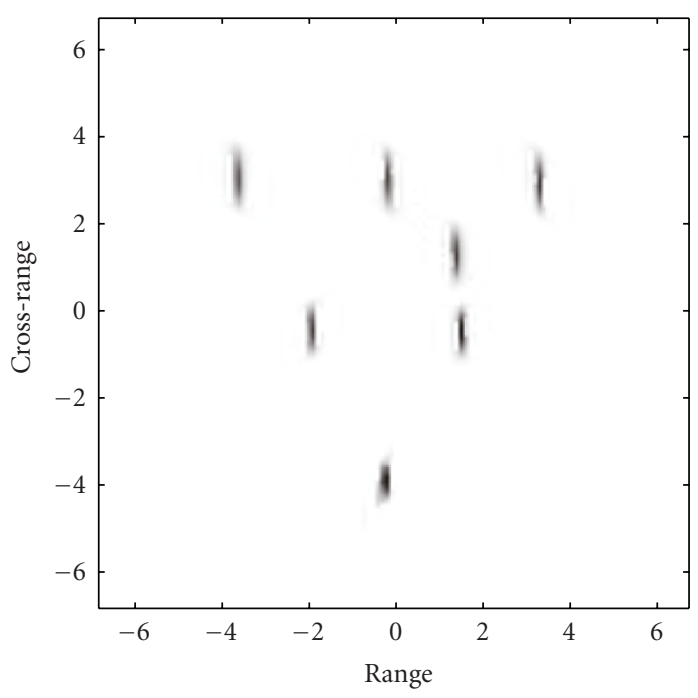

(c)

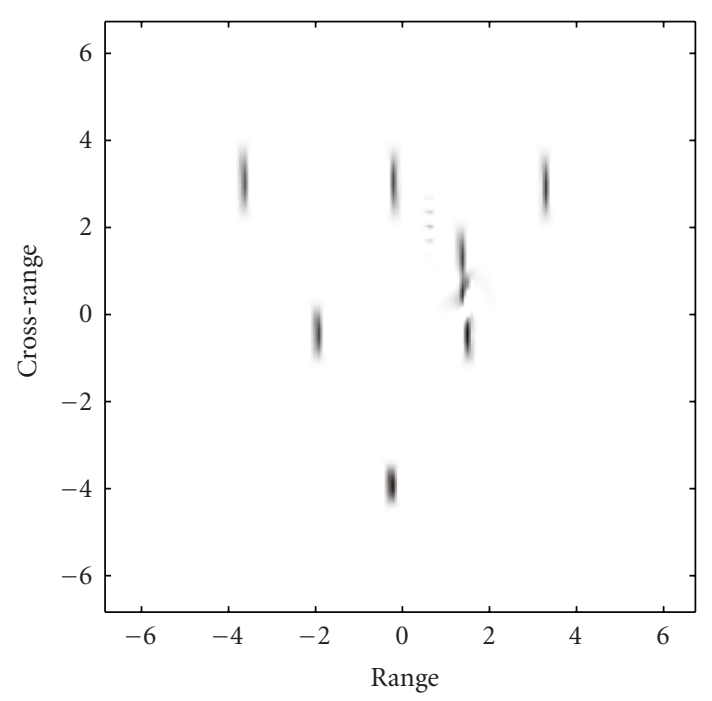

(b)

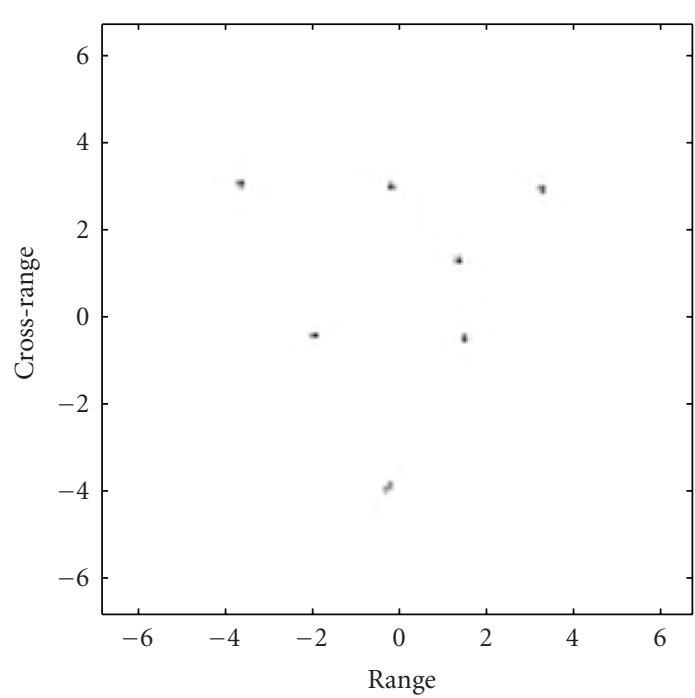

(d)

FIgURe 3: Simulated ISAR image obtained by using (a) 2D FT, (b) $1 \mathrm{D}$ SM with $K=8$, (c) adaptive 1D SM, and (d) adaptive 2D SM.

using information from both range and cross-range directions, the adaptive 2D SM is applied and the resulting radar image is shown in Figure 3(d). The resulting radar image is better concentrated and without undesired cross-terms. The threshold obtained by performing the previously described Otsu algorithm-based procedure is used for the adaptive 2D SM and adaptive 1D SM calculation.

\subsection{Application to the MIG target model}

The advantage of the adaptive 1D SM is illustrated on the MIG target model. This model is commonly used as a standard benchmark for comparison of different ISAR imaging methods. The ISAR image of the MIG obtained by using the 2D FT is shown in Figure 4(a), while the ISAR im- age obtained after applying the SM with $K=6$ is given in Figure 4(b). The points on the nose of the MIG and the upper part of the wings are blurred in the ISAR image obtained by using the 2D FT as a result of maneuvers they perform. The points on the tail of the MIG and lower part of the wings are well concentrated and no additional focusing is necessary. After the SM is applied to the radar signal, the concentration of the target's points which belong to the nose and upper part of the wings is improved, but cross-terms appear between the close points on the tail of the MIG and lower part of the wings. In Figure 4(c), the ISAR image of the MIG obtained by using the adaptive 1D SM is shown. The image is more focused and with reduced number of cross-terms compared to the radar image obtained by using the SM. For the adaptive $1 \mathrm{D}$ SM, $3 \%$ of the maximal value of the $2 \mathrm{D}$ FT-based radar image is used as threshold. 


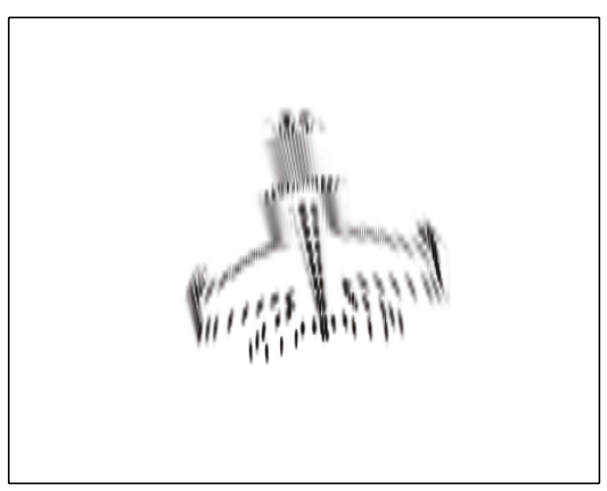

(a)

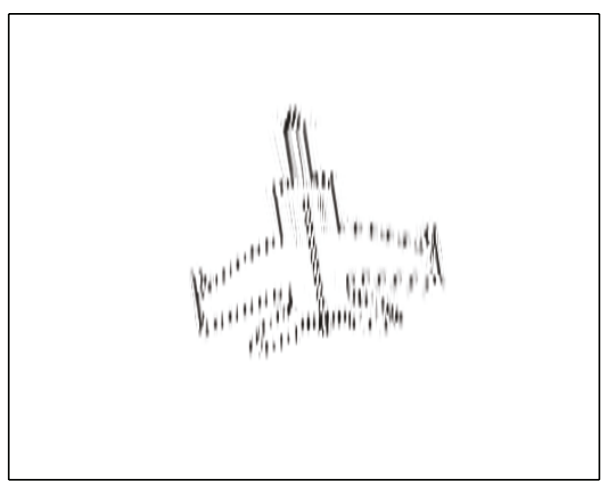

(b)

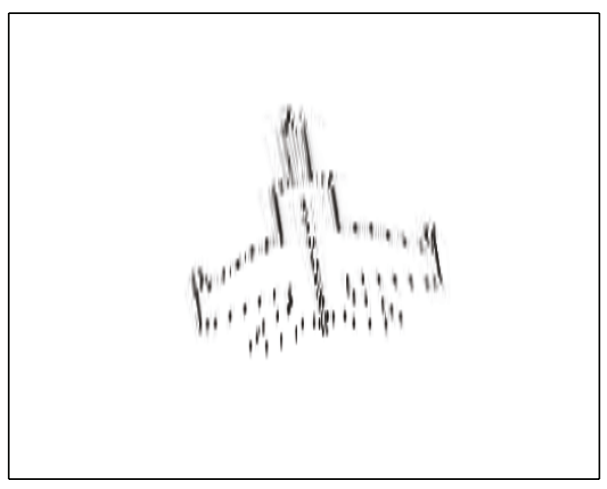

(c)

FIGURE 4: ISAR image of MIG obtained by using (a) 2D FT, (b) 1D SM with $K=6$, and (c) adaptive 1D SM.

\section{CONCLUSION}

An efficient adaptive technique for postprocessing SAR/ISAR images obtained using 2D FT is proposed. This technique is based on the adaptive 1D and 2D SM. It has been shown that simple strategy for adaptive selection of the frequency window width in the SM produces excellent results with highly focused radar images and with avoiding undesired interference terms. The adaptive selection of frequency window width is an important part of the proposed technique. The threshold determination issue is discussed and the procedure based on the Otsu algorithm appears to be very efficient. Numerical examples confirm quality of the proposed technique.

\section{REFERENCES}

[1] V. C. Chen and H. Ling, Time-Frequency Transforms for Radar Imaging and Signal Analysis, Artech House, Boston, Mass, USA, 2002.

[2] W. G. Carrara, R. S. Goodman, and R. M. Majewski, Spotlight Synthetic Aperture Radar-Signal Processing Algorithms, Artech House, Norwood, Ohio, USA, 1995.

[3] S. Wong, E. Riseborough, and G. Duff, "Experimental investigation on the distortion of ISAR images using different radar waveforms," Technical Memorandum TM 2003-196, Defence Research and Development Canada, Ottawa, Canada, 2003.

[4] S. R. DeGraaf, "SAR imaging via modern 2-D spectral estimation methods," IEEE Transactions on Image Processing, vol. 7, no. 5, pp. 729-761, 1998.

[5] Z. S. Lieu, R. Wu, and J. Li, "Complex ISAR imaging of maneuvering targets via the Capon estimator," IEEE Transactions on Signal Processing, vol. 47, no. 5, pp. 1262-1271, 1999.

[6] V. C. Chen and W. J. Miceli, "Simulation of ISAR imaging of moving targets," IEE Proceedings: Radar, Sonar and Navigation, vol. 148, no. 3, pp. 160-166, 2001.

[7] R. K. Raney, "Synthetic aperture imaging radar and moving targets," IEEE Transactions on Aerospace and Electronic Systems, vol. 7, no. 3, pp. 499-505, 1971.

[8] M. Kirscht, "Detection and imaging of arbitrarily moving targets with single-channel SAR," IEE Proceedings: Radar, Sonar and Navigation, vol. 150, no. 1, pp. 7-11, 2003.

[9] V. C. Chen, F. Li, S.-S. Ho, and H. Wechsler, "Analysis of microDoppler signatures," IEE Proceedings: Radar, Sonar and Navigation, vol. 150, no. 4, pp. 271-276, 2003.

[10] T. Sparr and B. Krane, "Micro-Doppler analysis of vibrating targets in SAR," IEE Proceedings: Radar, Sonar and Navigation, vol. 150, no. 4, pp. 277-283, 2003.

[11] I. Djurović, T. Thayaparan, and L. Stanković, "Adaptive local polynomial Fourier transform in ISAR," EURASIP Journal on Applied Signal Processing, vol. 2006, Article ID 36093, 15 pages, 2006.

[12] H. L. Chan and T. S. Yeo, "Comments on non-iterative quality phase-gradient autofocus (QPGA) algorithm for spotlight SAR imagery," IEEE Transactions on Geoscience and Remote Sensing, vol. 40, no. 11, p. 2517, 2002.

[13] H. L. Chan and T. S. Yeo, "Noniterative quality phase-gradient autofocus (QPGA) algorithm for spotlight sar imagery," IEEE Transactions on Geoscience and Remote Sensing, vol. 36, no. 5, part 1, pp. 1531-1539, 1998.

[14] W. Haiqing, D. Grenier, G. Y. Delisle, and F. Da-Gang, "Translational motion compensation in ISAR image processing," IEEE Transactions on Image Processing, vol. 4, no. 11, pp. 15611571, 1995.

[15] J. K. Jao, "Theory of synthetic aperture radar imaging of a moving target," IEEE Transactions on Geoscience and Remote Sensing, vol. 39, no. 9, pp. 1984-1992, 2001.

[16] Y. Wang, H. Ling, and V. C. Chen, "ISAR motion compensation via adaptive joint time-frequency techniques," IEEE Transactions on Aerospace and Electronics Systems, vol. 34, no. 2, pp. 670-677, 1998.

[17] L. Cohen, Time-Frequency Analysis, Prentice Hall, Upper Saddle River, NJ, USA, 1995.

[18] V. N. Ivanović, M. Daković, and LJ. Stanković, "Performance of quadratic timefrequency distributions as instantaneous frequency estimators," IEEE Transactions on Signal Processing, vol. 51, no. 1, pp. 77-89, 2003. 
[19] B. Boashash, "Estimating and interpreting the instantaneous frequency of a signal-Part 1," IEEE Proceedings, vol. 80, no. 4, pp. 519-538, 1992.

[20] LJ. Stanković, T. Thayaparan, M. Daković, and V. Popović, "Smethod in radar imaging," in Proceedings of the 14th European Signal Processing Conference (EUSIPCO '06), Florence, Italy, September 2006.

[21] S. Stanković and LJ. Stanković, "An architecture for the realization of a system for time-frequency signal analysis," IEEE Transactions on Circuits and Systems-Part II, no. 7, pp. 600604, 1997.

[22] R. C. Gonzalez and R. E. Woods, Digital Image Processing, Prentice Hall, Upper Saddle River, NJ, USA, 2002.

[23] V. N. Ivanović, R. Stojanović, and LJ. Stanković, "Multiple clock cycle architecture for the VLSI design of a system for time-frequency analysis," EURASIP Journal on Applied Signal Processing, vol. 2006, Article ID 60613, 18 pages, 2006.

[24] J. J. Sharma, C. H. Gierull, and M. J. Collins, "Compensating the effects of target acceleration in dual-channel SAR-GMTI," IEE Proceedings: Radar, Sonar and Navigation, vol. 153, no. 1, pp. 53-62, 2006.

[25] J. J. Sharma, C. H. Gierull, and M. J. Collins, "The influence of target acceleration on velocity estimation in dualchannel SAR-GMTI," IEEE Transactions on Geoscience and Remote Sensing, vol. 44, no. 1, pp. 134-147, 2006.

[26] LJ. Stanković, "A method for time-frequency analysis," IEEE Transactions on Signal Processing, vol. 42, pp. 225-229, 1994.

[27] L. Stanković, V. Ivanović, and Z. Petrović, "Unified approach to noise analysis in the Wigner distribution and spectrogram," Annales des Telecommunications/Annals of Telecommunications, vol. 51, no. 11-12, pp. 585-594, 1996.

[28] S. Stanković, I. Djurović, and V. Vuković, "System architecture for space-frequency image analysis," Electronics Letters, vol. 34, no. 23, pp. 2224-2225, 1998. 